\title{
Five-dimensional gauge theories from shifted web diagrams
}

\author{
Brice Bastian, ${ }^{1, *}$ Stefan Hohenegger, ${ }^{1, \dagger}$ Amer Iqbal, ${ }^{2,3, *}$ and Soo-Jong Rey ${ }^{4, \S}$ \\ ${ }^{1}$ Université de Lyon, UMR 5822, CNRS/IN2P3, Institut de Physique Nucléaire de Lyon, \\ 4 rue Enrico Fermi, 69622 Villeurbanne Cedex, France \\ ${ }^{2}$ Abdus Salam School of Mathematical Sciences, Government College University, Lahore 54600, Pakistan \\ ${ }^{3}$ Center for Theoretical Physics, Lahore 54600, Pakistan \\ ${ }^{4}$ School of Physics and Astronomy, Seoul National University, \\ Gwanak-ro 1, Gwanak-gu, Seoul 08826, Korea
}

(Received 3 November 2018; published 20 February 2019)

\begin{abstract}
In previous works [J. High Energy Phys. 07 (2017) 112; Phys. Rev. D 97, 046004 (2018); J. High Energy Phys. 11 (2018) 016], we studied a class of toric Calabi-Yau threefolds which engineer six-dimensional supersymmetric gauge theories with gauge group $U(N)$ and adjoint matter. The Kähler moduli space of these manifolds can be extended through flop transformations to include regions which are described by so-called shifted toric web diagrams. In this paper, we analyze gauge theories that are engineered by these shifted toric web diagrams and argue that, in specific limits, some of them engineer five-dimensional quiver gauge theories with gauge group $G \subset U(N)$ and with fundamental and bifundamental matter. We discuss several examples in detail and describe how the matter sector is obtained from the six-dimensional parent theory.
\end{abstract}

DOI: $10.1103 /$ PhysRevD.99.046012

\section{INTRODUCTION}

The engineering of five- and six-dimensional superconformal theories in string theory, using branes or geometry, has provided insights into various properties of these theories including the allowed matter content. More recently, the classification of the five- and sixdimensional superconformal theories has been studied using $\mathrm{M}$ and $\mathrm{F}$ theory on Calabi-Yau threefolds [1-5]. An extension of the classification of six-dimensional superconformal theories also resulted in the classification of little string theories [6]. The latter have an intrinsic scale set by $M_{\text {string }}$ and are related to superconformal theories by a particular limit which gets rid of this scale [7-15]. The geometry of Calabi-Yau threefolds plays an important role in these classifications determining the gauge groups and matter content of the available theories [16-19].

In this paper, we continue our study of a particularly rich class of such Calabi-Yau threefolds, called $X_{N, M}$ (with $N$, $M \in \mathbb{N}$ ), which was introduced in [20-22]. These manifolds are toric [23] and are parametrized by $N M+2$ independent

\footnotetext{
*b.bastian@ipnl.in2p3.fr

†s.hohenegger@ipnl.in2p3.fr

*amer@alum.mit.edu

rey.soojong@gmail.com
}

Published by the American Physical Society under the terms of the Creative Commons Attribution 4.0 International license. Further distribution of this work must maintain attribution to the author(s) and the published article's title, journal citation, and DOI. Funded by SCOAP.
Kähler parameters. It was proposed in [24] that a given $X_{N, M}$ engineers via $\mathrm{F}$ theory three different quiver gauge theories in six dimensions (compactified on $S^{1}$ ) with gauge groups $[U(N)]^{M},[U(M)]^{N}$, and $[U(N M / k)]^{k}$, respectively, where $k=\operatorname{gcd}(N, M)$. These theories are dual to each other in the sense that they have the same partition function $\mathcal{Z}_{N, M}(\{\mathbf{h}, \mathbf{v}, \mathbf{m}\})$, where $\{\mathbf{h}, \mathbf{v}, \mathbf{m}\}$ is the set of Kähler parameters of $X_{N, M}$ (with a suitable parametrization). The main difference between the three gauge theories is the interpretation of these Kähler parameters in terms of the gauge theory data, namely, coupling constants, and the mass parameters of the matter content as well as parameters related to the gauge structure.

It was further pointed out in [25] that the number of (different) dual gauge theories is, in general, even higher than three. Indeed, it was argued in [24] [and demonstrated explicitly in [26] for $\operatorname{gcd}(N, M)=1$ ] that the Calabi-Yau manifolds $X_{N, M}$ and $X_{N^{\prime}, M^{\prime}}$ [with $N M=N^{\prime} M^{\prime}$ and $\left.\operatorname{gcd}(N, M)=\operatorname{gcd}\left(N^{\prime}, M^{\prime}\right)\right]$ are related through a series of flop transformations that leave $\mathcal{Z}_{N, M}(\{\mathbf{h}, \mathbf{v}, \mathbf{m}\})$ invariant. This implies that $X_{N . M}$ engineers a web of dual gauge theories with gauge groups $\left[U\left(N^{\prime}\right)\right]^{M^{\prime}}$ for all $\left(M^{\prime}, N^{\prime}\right)$ satisfying the above conditions.

The duality transformation discovered in [24] is particular in the sense that it relates specific classes of toric web diagrams to one another. However, the extended moduli space [16,19,27-31] of $X_{N, M}$ also contains other geometries, such as the so-called shifted webs $X_{N, M}^{(\delta)}$, which were introduced in [25]. The latter are parametrized by an integer $\delta \in\{0,1, \ldots, N-1\}$, which governs how the planar web is 


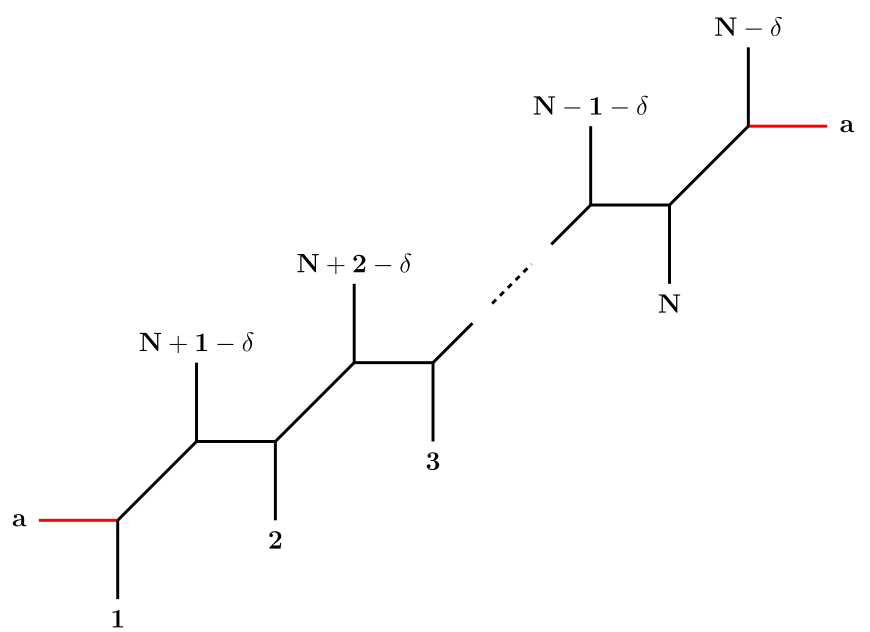

FIG. 1. Web diagram for $X_{N, 1}^{(\delta)}$. The labeling of the lines at the top of the diagram is understood to be modulo $N$, such that for $\delta=0$ we get back the "usual" web diagram $X_{N, 1}$.

glued together on a torus (an example for $M=1$ is shown in Fig. 1), such that $X_{N, M}^{(\delta=0)}=X_{N, M}$. While for $\delta=0$ F-theory compactifications on $X_{N, M}^{(\delta=0)}$ are dual to M5-brane configurations probing a nontrivial background, a similar formulation for the cases $\delta \neq 0$ is more delicate. In particular, while a straightforward decompactification limit exists in the former case, a similar limit is not immediately obvious in the latter case. This also makes the question what gauge theories (if any) are engineered by the configurations $X_{N, M}^{(\delta \neq 0)}$ more difficult to answer, since a simple five-dimensional limit (in which the partition function $\mathcal{Z}_{N, M}$ could be compared with the Nekrasov partition function) is not evident. In this paper, we analyze this problem for the case of shifted web diagrams of the form $X_{N, 1}^{(\delta)}$. We show first, with the help of a number of examples, that such web diagrams allow nontrivial oneparameter limits which reduce them to configurations that are known to engineer five-dimensional quiver gauge theories. While the gauge group of the latter is, in general, a subgroup of $U(N)$, they generically exhibit a rich matter spectrum. Based on these examples, we can generalize our discussion by extending a recurrent pattern.

Specifically, this paper is organized as follows: In Sec. II, we study various examples of the decompactification of shifted webs which reduce the six-dimensional theory to a five-dimensional theory. Different decompactification limits give rise to various different five-dimensional theories which can be thought of as dual to one another once lifted back up to six dimensions. We particularly point out that certain well-known five-dimensional theories arise via the decompactification of manifolds that are described by the above-mentioned shifted web diagrams. Generalizing a pattern appearing in the examples of Sec. II, we propose a two-parameter series of five-dimensional theories in
Sec. III, which arises from the decompactification limit of shifted web diagrams of the type $X_{A+B+A B, 1}^{(B(A+1)-1)}$ for $A$, $B \in \mathbb{N}$ (subject to certain conditions). In Sec. IV, we present our conclusions and comment on future directions. Furthermore, several technical details have been relegated to two Appendixes.

\section{SPECIFIC EXAMPLES}

The two-parameter class of Calabi-Yau threefolds (labeled interchangeably $X_{N, M}$ or $X_{N, M}^{(\delta=0)}$ in the current work) that was studied in $[24,26,32,33]$ is dual to $N$ M5-branes wrapped on $S^{1}$ probing a transverse almost locally Euclidean space of type $\hat{A}_{M-1}$. The Kähler moduli space of these manifolds takes the form of a cone which can be extended through flop transitions of $(-1,-1)$ curves $^{1}$ in $X_{N, M}$. In [24] it was argued (and reviewed in [26]) that a particular sequence of such transformations allows one to connect the Kähler cones of $X_{N, M}^{(\delta=0)}$ and $X_{N^{\prime}, M^{\prime}}^{(\delta=0)}$ if $N M=$ $N^{\prime} M^{\prime}$ and $\operatorname{gcd}(N, M)=\operatorname{gcd}\left(N^{\prime}, M^{\prime}\right){ }^{2}$ The Kähler cones of $X_{N, M}$ and $X_{N^{\prime}, M^{\prime}}$ are thus conjectured to be part of a larger, so-called extended Kähler moduli space. In [26], this conjecture was explicitly verified at the level of the topological string partition function $\mathcal{Z}_{N, M}$ of $X_{N, M}$ for the case of $\operatorname{gcd}(N, M)=1$. The partition function takes the schematic form

$\mathcal{Z}_{N, M}=\sum_{\alpha}\left(\prod_{i=1}^{M} \prod_{j=1}^{N} e^{-u_{i j}\left|\alpha_{j}^{i}\right|}\right) \prod_{j=1}^{N} W_{\alpha_{j+1}^{1} \ldots \alpha_{j+1}^{M}}^{\alpha_{j}^{1} \ldots \alpha_{j}^{M}}\left(Q_{\text {Kähler }}\right)$,

where $W_{\alpha_{j+1}^{1} \ldots \alpha_{j+1}^{M}}^{\alpha_{j}^{1} \ldots \alpha_{j}^{M}}$ are a set of universal building blocks, which are labeled by $N M$ integer partitions $\alpha_{j}^{i}$ (with $\left.\alpha_{j+N}^{i}=\alpha_{j}^{i}\right)$ and $\left(u_{i j}, Q_{\text {Kähler }}\right)$ are a particular parametrization of the Kähler parameters of $X_{N, M}$. For more details (specifically, the precise dependence of $W_{\alpha_{j+1}^{1} \ldots \alpha_{j+1}^{M}}^{\alpha_{j}^{1} \ldots \alpha_{j}^{M}}$ on $Q_{\text {Kähler}}$ ), as well as an interpretation of the latter from a gauge theoretical point of view, we refer the reader to $[25,26]$ (for the discussion of the topological string partition function of elliptic Calabi-Yau threefolds, see [34,35]). We remark, however, that $\mathcal{Z}_{N, M}$ transforms covariantly with respect to two copies of $S L(2, \mathbb{Z})$, one of which is made manifest in the formulation (2.1). The modular parameter of the latter shall be denoted $\rho$ in the following.

\footnotetext{
${ }^{1}$ We denote curves with local geometry $\mathcal{O}(a) \oplus \mathcal{O}(b) \mapsto \mathbb{P}^{1}$ as $(a, b)$ curves, where $a+b=-2$ due to the Calabi-Yau condition.

${ }^{2}$ It was argued in [25] that these relations imply the existence of an unexpectedly large web of dualities between the sixdimensional gauge theories that are engineered by these web diagrams. More precisely, it was conjectured that theories with gauge group $[U(N)]^{M}$ are dual to those with $\left[U\left(N^{\prime}\right)\right]^{M^{\prime}}$, where $(N, M)$ and $\left(N^{\prime}, M^{\prime}\right)$ are related as above.
} 
The toric manifolds $X_{N, M}$ engineer six-dimensional gauge theories compactified on a circle $S^{1}$ with radius $R$, which (in suitable units) is related to $\rho$ as follows:

$$
\operatorname{Im}(\rho)=\frac{1}{R} .
$$

The five-dimensional limit $R \rightarrow 0$ thus corresponds to the limit $\rho \rightarrow i \infty$ [one of the cusps of the corresponding $S L(2, \mathbb{Z})]$, which is well defined from the perspective of (2.1) and can, in fact, be obtained through an expansion in terms of $Q_{\rho}=e^{2 \pi i \rho}$. Geometrically, the elliptic parameter $\rho$ is associated to an elliptic curve which can be realized as a sum of $N \mathbb{P}^{1}$ 's. Sending $\rho \rightarrow i \infty$ is achieved by taking the size of one of these $\mathbb{P}^{1}$ 's to infinity. The result is still a toric Calabi-Yau manifold which can thus be used to engineer a five-dimensional gauge theory.

During intermediate steps of the previously mentioned sequence of flop transformations discussed in [24], other Kähler cones are encountered which can be described with so-called shifted web diagrams. An example of the latter [corresponding to $X_{N, 1}^{(\delta)}$ for $\left.\delta \in[0, N-1)\right]$ is shown in Fig. 1: The vertical lines at the top and the bottom of the diagram are glued together with a shift $\delta$. In fact, it was argued in [24,26] that a $X_{N, M}^{(\delta)}$ web diagram can be related to a $X_{N, M}^{(\delta+k)}$ web diagram [where $k=\operatorname{gcd}(N, M)$ ] through a combination of flop [and $S L(2, \mathbb{Z})$ ] transformations. A five-dimensional limit of the type $\rho \rightarrow i \infty$, as in the case of $\delta=0$ before, is difficult to realize for the shifted webs with $\delta \neq 0$. This can be directly understood from the diagram in Fig. 1: The naïve limit $\rho \rightarrow i \infty$ corresponds to pulling the two horizontal lines that are glued along the label $a$ (drawn in red in Fig. 1) off to infinity. In this limit (and for $\delta \neq 0$ ), however, the only way of gluing the lines at the bottom of the diagram to the ones at the top of the diagram involves crossings, which are not consistent. This, however, does not preclude the existence of other, more involved limits of the Kähler parameters of the web diagram $X_{N, M}^{(\delta)}$, which leave a consistent web diagram thus engineering a viable fivedimensional gauge theory. In the remainder of this section, we shall discuss with the help of a number of examples the existence of such novel limits and analyze the types of fivedimensional gauge theories they can give rise to. While the five-dimensional limit of theories with $\delta=0$, in general, leads to the same gauge groups as their six-dimensional counter parts, for $\delta \neq 0$, we find new gauge groups (which are subgroups of their six-dimensional parents), however, with a richer matter content.

\section{A. Example: $(N, M)=(3,1)$}

To illustrate the problem of finding an appropriate decompactification limit for nontrivial shift $\delta \neq 0 \bmod N$, we begin with the simplest nontrivial example, namely, $X_{3,1}^{(\delta=1)}$, whose web diagram is shown in Fig. 2 along with a

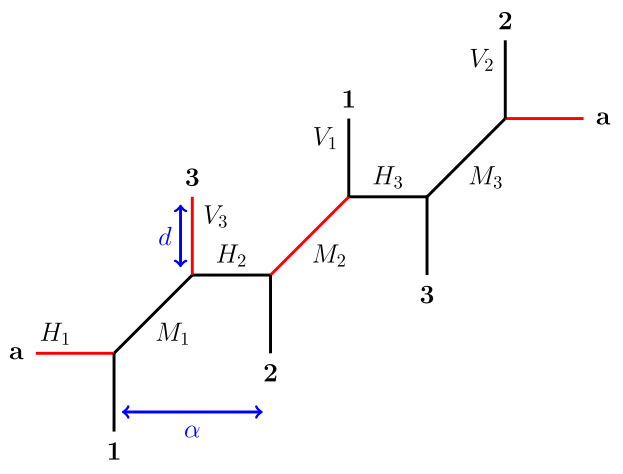

FIG. 2. Web diagram of $X_{3,1}^{(1)}$.

labeling of all the Kähler parameters $\left(H_{1,2,3}, V_{1,2,3}, M_{1,2,3}\right)$ (the blue parameters, as well as the significance of the red lines, shall be discussed below). Since the vertical curves at the top of the diagram are identified with their counterparts at the bottom with the relative shift $\delta=1$, the limit $H_{1} \rightarrow \infty$ cannot be taken in a way such that none of the remaining lines intersect one another. However, as we shall discuss now, there is another limit which leads to a noncompact web diagram that engineers a five-dimensional gauge theory. To understand how to obtain this limit, we consider the geometry of $\mathbb{F}_{1} \cup \mathbb{F}_{1}$ compactified on a torus (i.e., with the external legs identified pairwise), as shown in Fig. 3 (see [36] for the noncompact case). The latter can be related to the web diagram of $X_{3,1}^{(\delta=1)}$ in Fig. 2 upon performing a flop transformation of the curves $-E_{1}$ and $-E_{2}$. To see this, we consider the presentation of the geometry in Fig. 3(b) and perform a flop transformation on the curve $-E_{1}$ to obtain the configuration shown in Fig. 4(a). After an $S L(2, \mathbb{Z})$ transformation, the web diagram takes the form shown in Fig. 4(b). Finally, performing a flop transformation of the curve $-E_{2}$, we obtain the geometry shown in Fig. 2 corresponding to the web diagram of $X_{3,1}^{(\delta=1)}$. Here the Kähler parameters are identified:

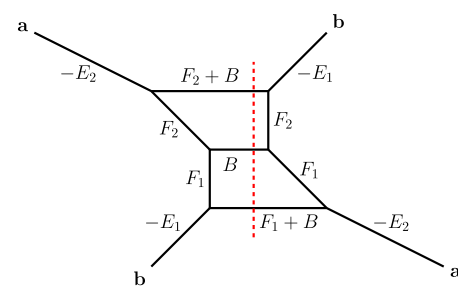

(a)

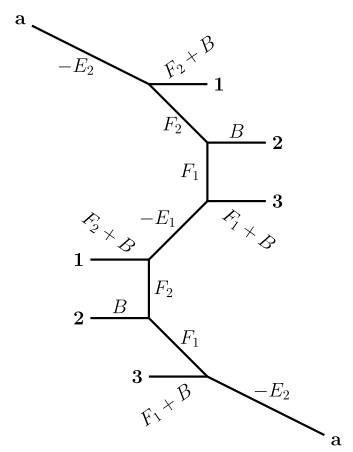

(b)
FIG. 3. (a) Gluing two copies of $\mathbb{F}_{1}$. (b) The same geometry after cutting along the red line and regluing along the line labeled $-E_{1}$. 


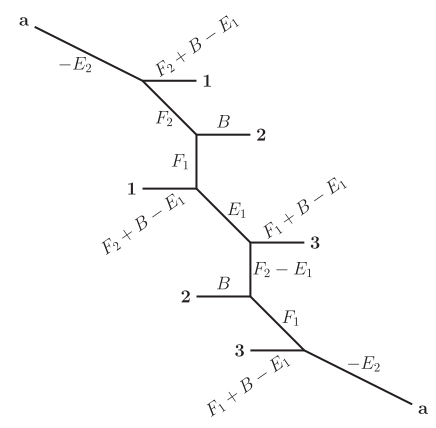

(a)

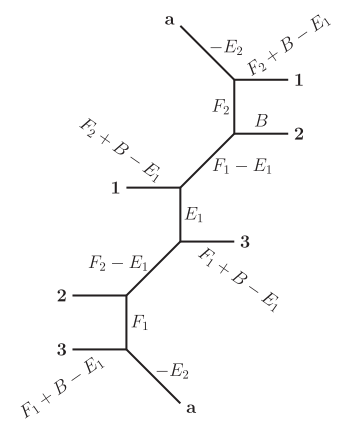

(b)
FIG. 4. (a) Geometry of Fig. 3 after a flop transformation of the line $-E_{1}$. (b) The same geometry after an $S L(2, \mathbb{Z})$ transformation.

$H_{1}=F_{2}-E_{2}, \quad H_{2}=E_{1}, \quad H_{3}=F_{1}-E_{2}$,

$V_{1}=B, \quad V_{2}=F_{1}+B-E_{1}-E_{2}, \quad V_{3}=F_{2}+B-E_{1}-E_{2}$, $M_{1}=F_{1}-E_{1}, \quad M_{2}=F_{2}-E_{1}, \quad M_{3}=E_{2}$.

From the relation between Figs. 3(a) and 2, we can get an inspiration for a five-dimensional limit of $X_{3,1}^{(1)}$. Indeed, Fig. 3(a) allows various geometrically nontrivial limits, some of which carry over to decompactifications of $X_{3,1}^{(\delta=1)}$ that do not have the problem of crossing or intersecting lines. One example is the limit $F_{2} \rightarrow \infty,{ }^{3}$ which in Fig. 2 corresponds to $H_{1}, V_{3}, M_{2} \rightarrow \infty$, whose web diagram is shown in Fig. 5. Here the label $\varnothing$ on the external legs indicates that these lines extend to infinity. To study the five-dimensional theory associated with this web in more detail (in particular, its matter content and partition function) in detail, it is convenient to introduce a basis of parameters $\left(d, a, g, m_{1}, m_{2}\right)$

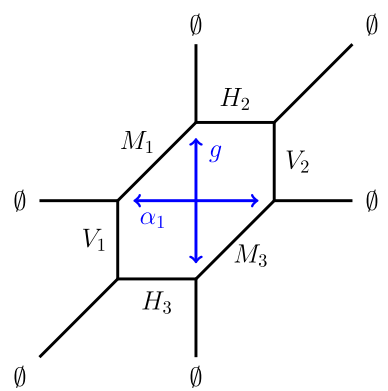

FIG. 5. Decompactified web diagram of $X_{3,1}^{(1)}$.

(partially shown in Fig. 2), such that all consistency conditions are satisfied:

$$
\begin{aligned}
2 a & =\alpha_{1}=H_{2}+M_{1}, \quad g=V_{1}+M_{1}, \quad d=V_{3}, \\
2 m_{1} & =M_{1}-H_{2}, \quad 2 m_{2}=M_{3}-H_{3} .
\end{aligned}
$$

The relation to the Kähler parameters appearing in Fig. 3 is

$F_{1}=2 a, \quad F_{2}=d+3 a-g+m_{2}, \quad B=g-a-m_{1}$,

$E_{1}=a-m_{1}, \quad E_{2}=a+m_{2}$,

while in terms of the parameters in Fig. 2 we have

$$
\begin{aligned}
& H_{1}=2 a+d-g, \quad H_{2}=a-m_{1}, \\
& H_{3}=a-m_{2}, \quad M_{1}=a+m_{1}, \\
& M_{2}=2 a+d-g+m_{1}+m_{2}, \quad M_{3}=a+m_{2}, \\
& V_{1}=g-m_{1}-a, \quad V_{2}=g-a-m_{2}, \quad V_{3}=d .
\end{aligned}
$$

Using this parametrization, the partition function $\mathcal{Z}_{3,1}^{(\delta=1)}$ takes the following form:

$$
\begin{aligned}
\mathcal{Z}_{3,1}^{(\delta=1)}= & \sum_{\alpha_{1}, \alpha_{2}, \alpha_{3}}\left(Q_{d} Q_{a} Q_{m_{1}}^{1 / 2} Q_{m_{2}}^{3 / 2}\right)^{\left|\alpha_{1}\right|}\left(Q_{d} Q_{a}^{3} Q_{m_{1}}^{1 / 2} Q_{m_{2}}^{3 / 2}\right)^{\left|\alpha_{2}\right|}\left(Q_{a}^{-2} Q_{m_{1}} Q_{m_{2}} Q_{g}\right)^{\left|\alpha_{3}\right|} \\
& \times \frac{\vartheta_{\alpha_{1} \alpha_{1}}(\mathcal{Q} ; \rho) \vartheta_{\alpha_{2} \alpha_{2}}(\mathcal{Q} ; \rho) \vartheta_{\alpha_{3} \alpha_{3}}(\mathcal{Q} ; \rho) \vartheta_{\alpha_{1} \alpha_{2}}\left(\mathcal{Q} Q_{a}^{-2} ; \rho\right) \vartheta_{\alpha_{2} \alpha_{1}}\left(\mathcal{Q} Q_{a}^{2} ; \rho\right)}{\vartheta_{\alpha_{3} \alpha_{3}}(1 ; \rho) \vartheta_{\alpha_{2} \alpha_{3}}\left(\mathcal{Q}^{-1} Q_{a} Q_{m_{2}} ; \rho\right) \vartheta_{\alpha_{1} \alpha_{3}}\left(\mathcal{Q}^{-1} Q_{a}^{-1} Q_{m_{2}} ; \rho\right) \vartheta_{\alpha_{3} \alpha_{2}}\left(\mathcal{Q} Q_{a}^{-1} Q_{m_{2}}^{-1} ; \rho\right)} \\
& \times \frac{\vartheta_{\alpha_{2} \alpha_{3}}\left(Q_{a} Q_{m_{2}} ; \rho\right) \vartheta_{\alpha_{1} \alpha_{3}}\left(Q_{m_{2}} Q_{a}^{-1} ; \rho\right) \vartheta_{\alpha_{3} \alpha_{2}}\left(Q_{m_{1}} Q_{a}^{-1} ; \rho\right) \vartheta_{\alpha_{3} \alpha_{1}}\left(Q_{m_{1}} Q_{a} ; \rho\right)}{\vartheta_{\alpha_{3} \alpha_{1}}\left(\mathcal{Q} Q_{a} Q_{m_{2}}^{-1} ; \rho\right) \vartheta_{\alpha_{1} \alpha_{1}}(1 ; \rho) \vartheta_{\alpha_{2} \alpha_{2}}(1 ; \rho) \vartheta_{\alpha_{1} \alpha_{2}}\left(Q_{a}^{-2} ; \rho\right) \vartheta_{\alpha_{2} \alpha_{1}}\left(Q_{a}^{2} ; \rho\right)},
\end{aligned}
$$

where the modular parameter is $\rho=8 a+2 d-2 g+$ $m_{1}+m_{2}$, we used the notation

$$
\begin{aligned}
Q_{a} & =e^{-a}, & Q_{d}=e^{-d}, \quad Q_{g}=e^{-g}, \\
Q_{m_{1,2}} & =e^{-m_{1,2},} \quad & \mathcal{Q}=Q_{d} Q_{a}^{4} Q_{m_{1}} Q_{m_{2}} Q_{g}^{-1},
\end{aligned}
$$

and the summations $\alpha_{1}, \alpha_{2}$, and $\alpha_{3}$ are over integer partitions of $\left|\alpha_{1}\right|,\left|\alpha_{2}\right|$, and $\left|\alpha_{3}\right|$, respectively. For the notation of the functions $\vartheta_{\mu \nu}$, we refer the reader to, e.g., [26].
In the limit $d \rightarrow \infty$, one has

$$
H_{1} \rightarrow \infty, \quad V_{3} \rightarrow \infty, \quad M_{2} \rightarrow \infty,
$$

which implies that the areas of the curves drawn in red in Fig. 2 become infinite and the corresponding lines are pulled to infinity. The remaining web diagram (shown in Fig. 5) is still toric and, in fact, corresponds to local $d P_{3}$.

\footnotetext{
${ }^{3}$ The latter corresponds to the large fiber limit of one of the $\mathbb{F}_{1}$ in Fig. 3(a).
} 
The latter engineers [36] a five-dimensional gauge theory with gauge group ${ }^{4} S U(2)$ and whose matter content transforms as $N_{f}=2$ copies of the fundamental representation. We denote this theory as $(S U(2), 2 \mathbf{F})$.

In the limit $d \rightarrow \infty$, also the partition function (2.6) simplifies, in particular, the theta functions can be written in terms of the Nekrasov factors ${ }^{5}$ as follows:

$$
\begin{aligned}
& \vartheta_{\mu \nu}(x ; \rho) \underset{\rho \rightarrow i \infty}{\longrightarrow} x^{-\frac{|\mu|+|\nu|}{2}} t^{\frac{1}{4}\left(\left\|\mu^{t}\right\|^{2}-\nu^{t} \|^{2}\right)} q^{-\frac{1}{4}\left(\|\mu\|^{2}-\|\nu\|^{2}\right)} N_{\mu \nu}\left(x \sqrt{\frac{t}{q}}\right), \\
& \vartheta_{\mu \nu}(x ; \rho) \underset{\rho \rightarrow i \infty, x \mapsto 0}{\longrightarrow} x^{-\frac{|\mu|+|\nu|}{2}} t^{\frac{1}{4}\left(\left\|\mu^{t}\right\|^{2}-\left\|\nu^{t}\right\|^{2}\right)} q^{-\frac{1}{4}\left(\|\mu\|^{2}-\nu \|^{2}\right)}
\end{aligned}
$$

where the Nekrasov factor is defined as

$$
\begin{aligned}
N_{\mu \nu}(x)= & \prod_{(i, j) \in \mu}\left(1-x t^{\nu_{j}^{t}-i} q^{\mu_{i}-j+1}\right) \\
& \times \prod_{(i, j) \in \nu}\left(1-x t^{-\mu_{j}^{t}+i-1} q^{-\nu_{i}+j}\right) .
\end{aligned}
$$

Using (2.8) in (2.6), the partition function takes the following form:

$\mathcal{Z}_{d P_{3}}^{5 D}=\sum_{\alpha_{1,2}}(-1)^{\left|\alpha_{1}\right|+\left|\alpha_{2}\right|}\left(Q_{a}^{-2} Q_{g} Q_{m_{1}}^{-1} Q_{m_{2}}^{-1}\right)^{\left|\alpha_{1}\right|+\left|\alpha_{2}\right|} \frac{N_{\alpha_{1} \varnothing}\left(Q_{m_{2}} Q_{a}^{-1} \sqrt{\frac{t}{q}}\right) N_{\varnothing \alpha_{1}}\left(Q_{m_{1}} Q_{a} \sqrt{\frac{t}{q}}\right) N_{\alpha_{2} \varnothing}\left(Q_{m_{2}} Q_{a} \sqrt{\frac{t}{q}}\right) N_{\varnothing \alpha_{2}}\left(Q_{m_{1}} Q_{a}^{-1} \sqrt{\frac{t}{q}}\right)}{N_{\alpha_{1} \alpha_{1}}\left(\sqrt{\frac{t}{q}}\right) N_{\alpha_{2} \alpha_{2}}\left(\sqrt{\frac{t}{q}}\right) N_{\alpha_{1} \alpha_{2}}\left(Q_{a}^{-2} \sqrt{\frac{t}{q}}\right) N_{\alpha_{2} \alpha_{1}}\left(Q_{a}^{2} \sqrt{\frac{t}{q}}\right)}$,

which is indeed the partition function of the five-dimensional $\mathcal{N}=1 S U(2)$ gauge theory with $N_{f}=2$ [37-40]. By associating weights to the $\vartheta$ functions in (2.6), one can see how the matter and vector representations get reduced upon taking the nontrivial five-dimensional limit discussed above. The weights are assigned based on the intersection numbers of the curves that make up the arguments with the compact divisors $S_{i}$ [41]. In Appendix B, we review a method to calculate the intersection numbers. Starting with the vector multiplet contribution [which gives rise to the $\vartheta$ functions in the denominator of the partition function in Eq. (2.6)], we have the following weight assignments in terms of Dynkin labels $\left(\left[\lambda_{1}\right],\left[\lambda_{2}\right]\right)$ of $\mathfrak{g} \mathfrak{t}(3)$ :

$$
\begin{aligned}
& \vartheta_{\alpha_{1} \alpha_{1}} \rightarrow[0,0], \quad \vartheta_{\alpha_{2} \alpha_{2}} \rightarrow[0,0], \quad \vartheta_{\alpha_{3} \alpha_{3}} \rightarrow[0,0], \\
& \vartheta_{\alpha_{1} \alpha_{2}} \rightarrow[2,-1], \quad \vartheta_{\alpha_{2} \alpha_{3}} \rightarrow[-1,2], \quad \vartheta_{\alpha_{1} \alpha_{3}} \rightarrow[1,1], \\
& \vartheta_{\alpha_{2} \alpha_{1}} \rightarrow[-2,1], \quad \vartheta_{\alpha_{3} \alpha_{2}} \rightarrow[-1,2],
\end{aligned}
$$

As expected for a vector multiplet, we find the $\mathfrak{s} \mathfrak{t}(3)$ adjoint representation plus a singlet. We can perform a similar analysis for the adjoint hypermultiplet contribution [which gives rise to the $\vartheta$ functions in the numerator of the partition function in Eq. (2.6)]:

$$
\begin{aligned}
& \vartheta_{\alpha_{1} \alpha_{1}} \rightarrow[0,0], \quad \vartheta_{\alpha_{2} \alpha_{2}} \rightarrow[0,0], \quad \vartheta_{\alpha_{3} \alpha_{3}} \rightarrow[0,0], \\
& \vartheta_{\alpha_{1} \alpha_{2}} \rightarrow[2,-1], \quad \vartheta_{\alpha_{2} \alpha_{3}} \rightarrow[-1,2], \quad \vartheta_{\alpha_{1} \alpha_{3}} \rightarrow[1,1], \\
& \vartheta_{\alpha_{2} \alpha_{1}} \rightarrow[-2,1], \quad \vartheta_{\alpha_{3} \alpha_{2}} \rightarrow[-1,2], \quad \vartheta_{\alpha_{3} \alpha_{1}} \rightarrow[-1,-1] .
\end{aligned}
$$

\footnotetext{
${ }^{4}$ There are no extra contributions to the partition function coming from parallel external legs [37]. Thus, the gauge group obtained is $S U(2)$ instead of $U(2)$.
}

Again, as expected, this gives the adjoint representation for the hypermultiplet plus a singlet. We can represent the vector and hypermultiplet representation in the weight lattices, as shown in Fig. 6. The weights that are colored in red are related to the $\vartheta$ functions whose argument goes to zero upon taking the $5 d$ limit. These weights get projected out, and we are left with an adjoint $\mathfrak{g} \mathfrak{t}(2)$ representation plus a singlet for the vector multiplet and two fundamental $\mathfrak{s} \mathfrak{t}(2)$ representations for the hypermultiplet. This agrees, as it should, with what one expects at the level of the web diagram.

\section{B. Example: $(N, M)=(5,1)$}

Similar decompactification limits as for the web diagram of $X_{3,1}^{(\delta=1)}$ discussed in the previous section can also be found for other configurations. As the next nontrivial example, we present the case $X_{5,1}^{(\delta)}$ for shifts $\delta=2$ and $\delta=3{ }^{6}$

\section{Shift $\delta=2$}

The web diagram of $X_{5,1}^{(2)}$ is shown in Fig. 7 along with a suitable labeling of the areas of all individual curves. Similar to the previous example, not all of the latter are independent, but they are subject to a number of consistency conditions, as explained in [21,42]. Inspired by the parametrization of $X_{3,1}^{(1)}$ above, we can introduce $\left(d, \alpha_{1}, \alpha_{2}, m_{1}, m_{2}, m_{3}, g\right)$ (as partially indicated in Fig. 7):

\footnotetext{
${ }^{5}$ For a partition $\mu=\left(\mu_{1}, \mu_{2}, \ldots\right)$ with parts $\mu_{i}$, we define $\|\mu\|^{2}=$ $\sum_{i} \mu_{i}^{2}$.

${ }^{6}$ The remaining shifts $\delta=1$ and $\delta=4$ can be directly related to these two by means of a simple rearrangement of the web diagram. They therefore do not give rise to new nontrivial limits.
} 


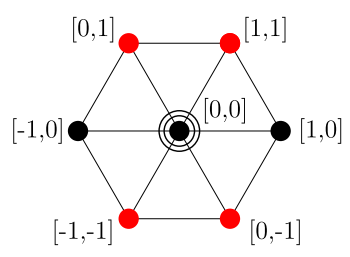

Vector representation

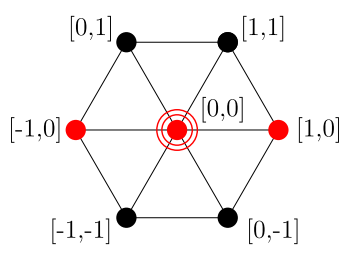

Matter representation

FIG. 6. The vector and matter representation of the $(3,1)$ web with shift $\delta=1$. The weights in red get "projected" out upon taking the $5 d$ limit. The circles around the weight $[0,0]$ indicate that the latter is threefold degenerate.

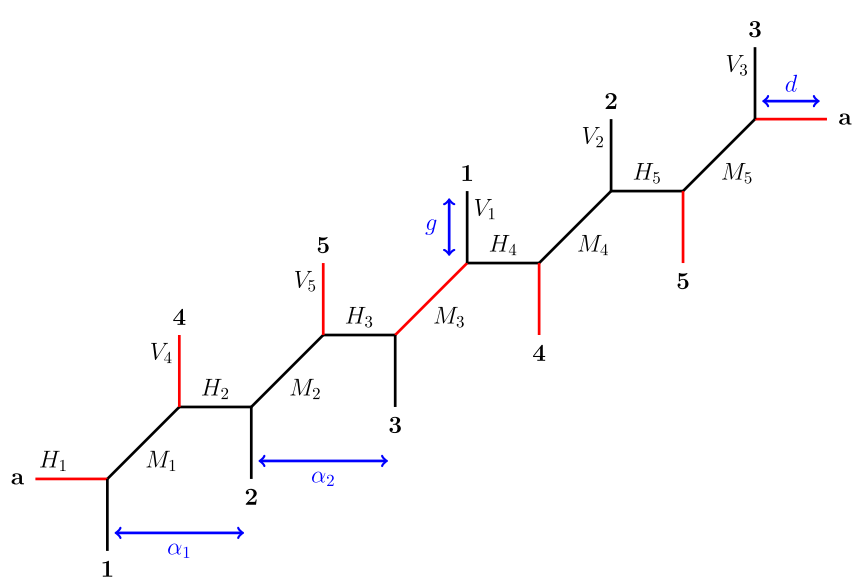

FIG. 7. Web diagram of $X_{5,1}^{(\delta=2)}$. The blue parameters provide a (partial) basis for labeling the areas for all curves of the diagram. The curves displayed in red are decompactified in the limit (2.15); i.e., their area is sent to infinity.

$$
\begin{aligned}
d & =H_{1}, \quad \alpha_{1}=M_{1}+H_{2}, \\
\alpha_{2} & =M_{2}+H_{3}, \quad m_{1}=M_{1}-H_{2}, \\
m_{2} & =M_{2}-H_{2}+M_{4}-H_{5}, \\
m_{3} & =M_{5}-H_{5}, \quad g=V_{2},
\end{aligned}
$$

which provide a solution to the consistency conditions of the form

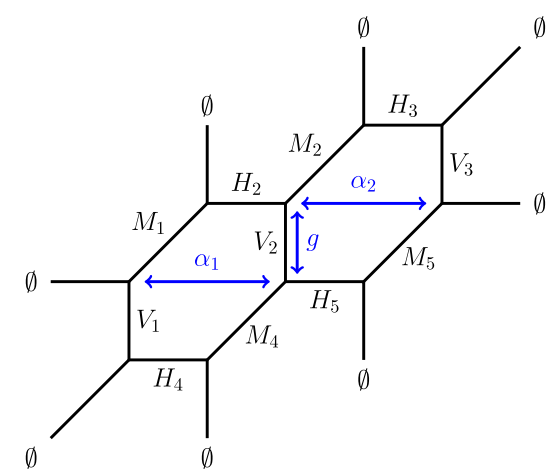

FIG. 8. Web diagram of the decompactified $X_{5,1}^{(\delta=2)}$.

$$
\begin{aligned}
& H_{1}=d, \quad H_{2}=\frac{\alpha_{1}-m_{1}}{2}, \quad H_{3}=\frac{\alpha_{2}-m_{2}+m_{3}}{2}, \\
& H_{4}=\frac{\alpha_{1}-m_{2}+m_{1}}{2}, \quad H_{5}=\frac{\alpha_{2}-m_{3}}{2}, \\
& V_{1}=\frac{2 g-2 m_{1}+m_{2}}{2}, \quad V_{2}=g, \quad V_{3}=\frac{2 g+m_{2}-2 m_{3}}{2}, \\
& V_{4}=\frac{2 d+2 g-m_{1}+2 m_{2}-2 m_{3}-\alpha_{1}}{2}, \\
& V_{5}=\frac{2 d+2 g-2 m_{1}+2 m_{2}-m_{3}-\alpha_{2}}{2}, \\
& M_{1}=\frac{m_{1}+\alpha_{1}}{2}, \quad M_{2}=\frac{m_{2}-m_{3}+\alpha_{2}}{2}, \quad M_{4}=\frac{\alpha_{1}-m_{1}+m_{2}}{2}, \quad M_{5}=\frac{\alpha_{2}}{2} . \\
& M_{3}=\frac{2 d+m_{2}}{2}, \quad M_{4} .
\end{aligned}
$$

In the limit $d \rightarrow \infty$ (while keeping the remaining parameters $\alpha_{1}, \alpha_{2}, m_{1}, m_{2}, m_{3}, g$ finite), we have

$$
H_{1} \rightarrow \infty, \quad V_{4,5} \rightarrow \infty, \quad M_{3} \rightarrow \infty
$$

The corresponding curves in the web diagram Fig. 7 (drawn in red) become noncompact, and the remaining web diagram can be presented in the form of Fig. 8. This geometry engineers a five-dimensional gauge theory, which has been previously studied in the literature [36]: It corresponds to a theory with gauge group $U(3)$ whose matter content transforms as four copies of its fundamental representation. Therefore, the limit $d \rightarrow \infty$ can be interpreted as a decompactification limit of the six-dimensional theory constructed from $X_{5,1}^{(\delta=2)}$ to five dimensions engineering the theory $(U(3), 4 \mathbf{F})$. However, it should be noted that the latter is not at a generic point in the moduli space: The theory $(U(3), 4 \mathbf{F})$ allows for seven independent parameters, ${ }^{7}$ while the theory engineered from the web diagram Fig. 8 has only six independent parameters (as a consequence of the consistency conditions of the compact diagram Fig. 7). As a consequence, the theory engineered in this manner has an additional constraint relating the masses of the matter fields.

\section{Shift $\delta=3$}

A limit similar to (2.15) can also be found for $X_{5,1}^{(\delta=3)}$, whose web diagram (along with a suitable labeling of the areas of the various curves) is shown in Fig. 9. Inspired by the case $\delta=2$, we introduce the set of parameters $\left(\alpha_{1}, \alpha_{2}\right.$, $\left.g_{1}, g_{2}, m_{1}, m_{2}, d\right)$, which are indirectly defined through

\footnotetext{
${ }^{7}$ These seven parameters correspond to a gauge coupling constant, two vacuum expectation values of vector multiplet scalars, and the masses of the four fundamental matter representations.
} 


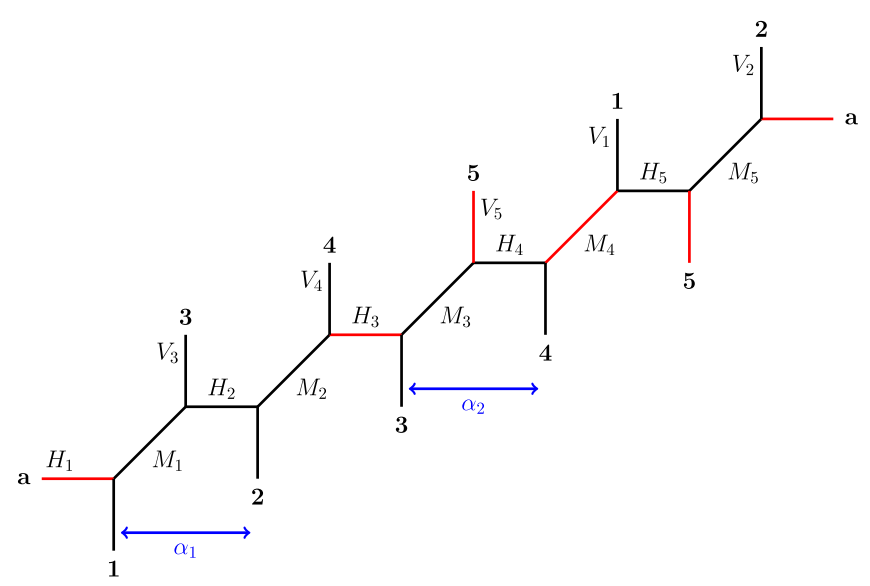

FIG. 9. Web diagram of $X_{5,1}^{(\delta=3)}$. The blue parameters provide a (partial) basis for labeling the areas for all curves of the diagram. The curves displayed in red become noncompact in the limit (2.18); i.e., their area is sent to infinity.

$$
\begin{aligned}
\alpha_{1} & =M_{1}+H_{2}, \quad \alpha_{2}=M_{2}+H_{2}, \quad H_{1}=d-\alpha_{1}, \\
g_{1} & =V_{1}+M_{1}, \quad g_{2}=M_{3}+V_{3}, \quad m_{1}=\alpha_{2}+M_{1}-H_{2}, \\
m_{2} & =g_{1}-g_{2}-H_{4}+M_{3}-2 \alpha_{1}+\alpha_{2} .
\end{aligned}
$$

These parameters provide a solution to the consistency conditions of the web diagram of $X_{5,1}^{(\delta=3)}$ in the sense that the areas of all curves $\left(H_{1, \ldots, 5}, V_{1, \ldots, 5}, M_{1, \ldots, 5}\right)$ can be expressed in terms of $\left(\alpha_{1}, \alpha_{2}, g_{1}, g_{2}, m_{1}, m_{2}, d\right)$ :

$$
\begin{aligned}
& H_{1}=d-\alpha_{1}, \quad H_{2}=\frac{\alpha_{1}+\alpha_{2}-m_{1}}{2}, \\
& H_{3}=d-\alpha_{2}, \quad H_{4}=\alpha_{2}-\alpha_{1}+\frac{g_{1}-g_{2}-m_{2}}{2}, \\
& H_{5}=\alpha_{1}-\alpha_{2}-\frac{g_{1}-g_{2}+m_{2}}{2}, \quad V_{1}=g_{1}-\frac{m_{1}+\alpha_{1}-\alpha_{2}}{2}, \\
& V_{2}=-\alpha_{2}+\frac{g_{1}+g_{2}-m_{2}}{2}, \quad V_{3}=-\alpha_{1}+\frac{g_{1}+g_{2}-m_{2}}{2} \\
& V_{4}=g_{2}-\frac{m_{1}-\alpha_{1}+\alpha_{2}}{2}, \\
& V_{5}=d+\frac{g_{1}+g_{2}-m_{1}+m_{2}-\alpha_{1}-\alpha_{2}}{2}, \\
& M_{1}=\frac{m_{1}+\alpha_{1}-\alpha_{2}}{2}, \quad M_{2}=\frac{m_{1}-\alpha_{1}+\alpha_{2}}{2} \\
& M_{3}=\alpha_{1}+\frac{m_{2}-g_{1}+g_{2}}{2}, \quad M_{4}=d+m_{2} \\
& M_{5}=\alpha_{2}+\frac{m_{2}+g_{1}-g_{2}}{2} .
\end{aligned}
$$

In the limit $d \rightarrow \infty$ [while keeping the remaining parameters $\left(\alpha_{1}, \alpha_{2}, g_{1}, g_{2}, m_{1}, m_{2}\right)$ finite], one finds

$$
H_{1}, H_{3} \rightarrow \infty, \quad V_{5} \rightarrow \infty, \quad M_{4} \rightarrow \infty,
$$

which means that the corresponding curves in the web diagram (indicated in red in Fig. 9) are decompactified.

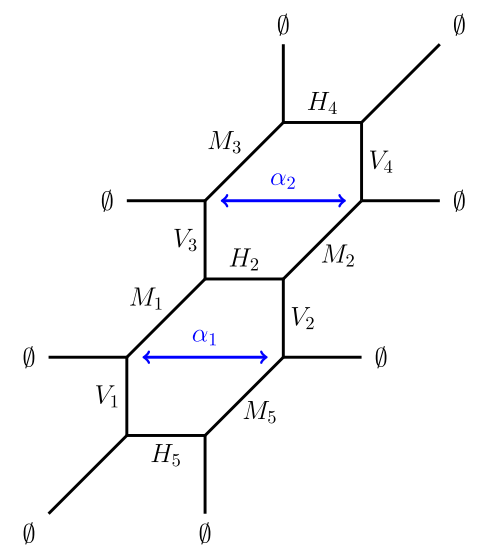

FIG. 10. Web diagram of the decompactified $X_{5,1}^{(\delta=3)}$.

The resulting diagram can be presented in the form of Fig. 10, where $\varnothing$ indicates that these curves are pulled all the way to infinity. Therefore, the diagram in Fig. 10 corresponds to a noncompact web, and the limit $d \rightarrow \infty$ can be understood as a decompactification limit to five dimensions. Furthermore, since Fig. 10 can be interpreted as an $S L(2, \mathbb{Z})$ transformation of Fig. 8, the five-dimensional gauge theory engineered by the former is, in fact, an S-dual version of $(U(3), 4 \mathbf{F})$, namely, $\left([U(2)]^{2}, 2 \mathbf{F}, \mathbf{B F}\right)$. The latter theory is indeed engineered by the same diagram Fig. 10 up to an $S L(2, \mathbb{Z})$ transformation (which acts essentially as a rotation).

Finally, similar to the case of $(U(3), 4 \mathbf{F})$ engineered from Fig. 8 , the $\left([U(2)]^{2}, 2 \mathbf{F}, \mathbf{B F}\right)$ theory engineered from Fig. 10 is not at a generic point in its moduli space. Instead, due to the consistency conditions of the original diagram Fig. 9, the theory is in a codimension-one subspace. The additional constraint relates the two coupling constants of the gauge groups.

\section{A TWO-PARAMETER SERIES OF FIVE-DIMENSIONAL THEORIES}

The examples of the previous section can be generalized to more sophisticated configurations: In the following, we shall provide nontrivial evidence that five-dimensional gauge theories of the type $\left([U(A+1)]^{B}, 2 A \mathbf{F},(B-1) \mathbf{B F}\right.$ ) (with $A, B \in \mathbb{N})$ can be engineered from a shifted web diagram.

Our starting point is the web diagram of $X_{A+B+A B, 1}^{(\delta=B(A+1)-1)}$, as shown in Fig. 11, where we have added a labeling of the areas of all curves. Here we assume $A>0$ and $B>0$. Web diagrams of this type are parametrized by $A+B+A B+2$ independent parameters. Generalizing the pattern of the previous examples, we conjecture that there exists a oneparameter limit such that

$$
\begin{aligned}
H_{1+k(A+1)} & \rightarrow \infty, \quad V_{B+A B+r} \rightarrow \infty, \\
M_{B+A B} & \rightarrow \infty \quad \forall\left\{\begin{array}{l}
k=0, \ldots, B-1, \\
r=1, \ldots, A
\end{array}\right.
\end{aligned}
$$




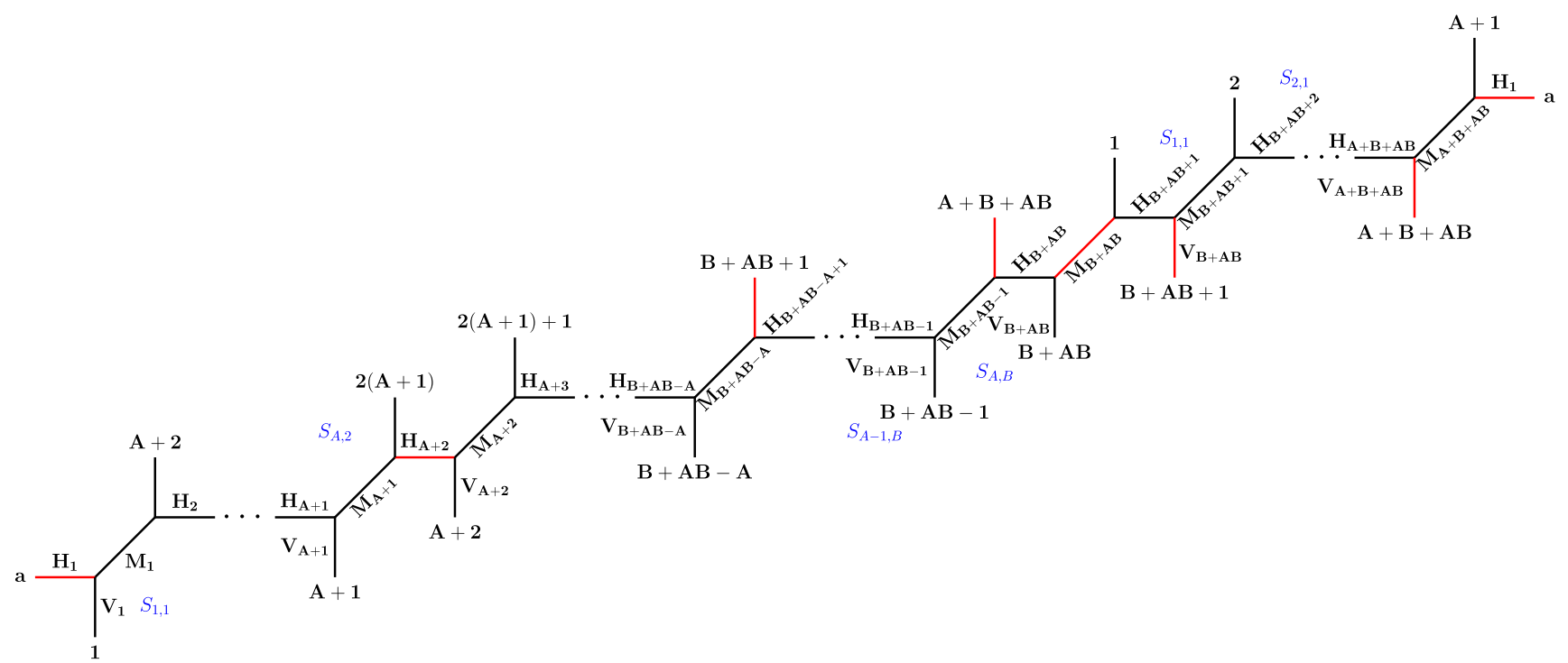

FIG. 11. Web diagram of $X_{A+B+A B, 1}^{(\delta=B(A+1)-1)}$. The curves drawn in red are being decompactified in the limit Eq. (3.1). The blue labels $S_{a, b}$ indicate the same hexagons as in Fig. 12.

while keeping the areas of all remaining curves finite. The $B$ horizontal, $A$ vertical, and single diagonal curve that are decompactified in the limit (3.1) are shown in red in Fig. 11. The resulting web diagram is noncompact and can be presented in the form of Fig. 12. The latter corresponds to $A B$ hexagons $S_{a, b}$ (with $a=1, \ldots, A$ and $b=1, \ldots, B)$ being arranged in a symmetric array.

The web diagram Fig. 12 engineers a (linear) quiver gauge theory in five dimensions with $B$ gauge nodes of

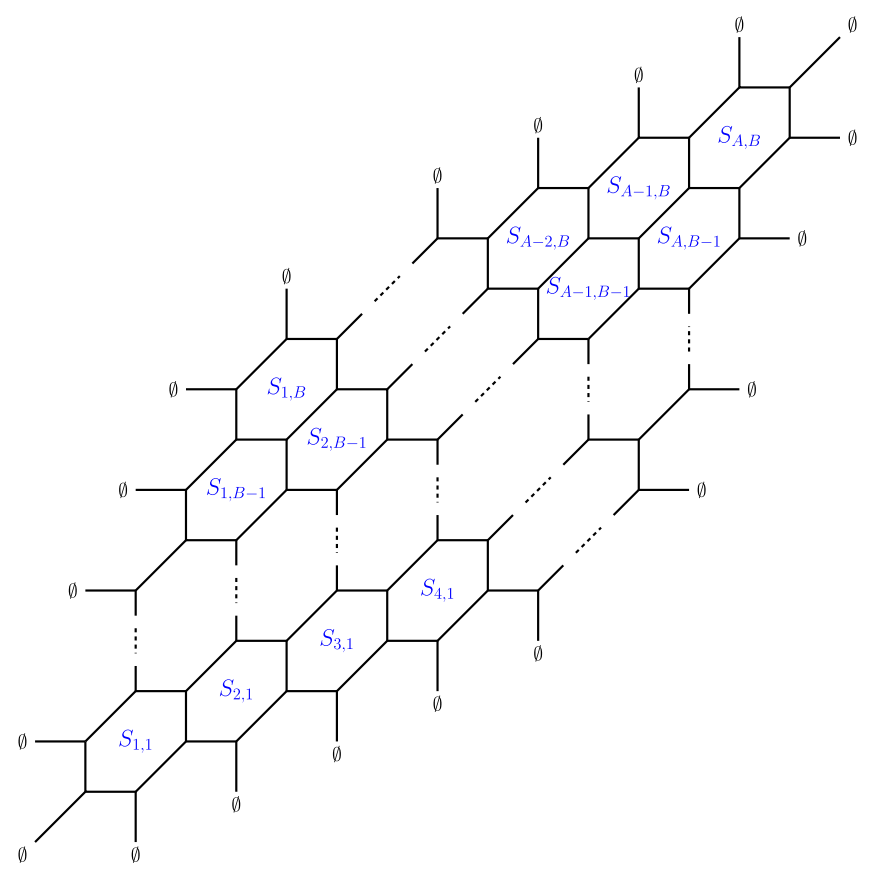

FIG. 12. Web diagram consisting of $A B$ hexagons obtained in the decompactification limit (3.1) from Fig. 11. gauge group $U(A+1)$, whose matter content consists of $B-1$ bifundamental representations and $N_{f}=2 A$ fundamental representations, which we denote by $\left([U(A+1)]^{B}, 2 A \mathbf{F},(B-1) \mathbf{B F}\right)$. The latter can be interpreted as the five-dimensional limit of the six-dimensional theory with gauge group $U(A+B+A B)$ and adjoint matter that is engineered from $X_{A+B+A B, 1}^{(\delta=B(A+1)-1)}$. It should, however, be noticed that the five-dimensional theory is not at a generic point in its moduli space: This can be seen by counting the independent parameters of the web diagrams. The web diagram in Fig. 11 has a maximal set of $A+B+$ $A B+2$ parameters, and the decompactification limit gets rid of one of them. As a consequence, we are left with $A+B+A B+1$ independent parameters in Fig. 12 . However, the most generic web of the type Fig. 12 allows for $A B+2(A+B)-1$ parameters. Hence, the theories we obtain in the reduction from six dimensions live in a codimension $A+B-2$ subspace of the full moduli space. For low values of $N=A+B+A B$, some of the five-dimensional theories that can be engineered as limits of shifted web diagrams are displayed in Table I. It should be noted that web diagrams of the same "length" $N$ (but different shift $\delta$ ) can give rise to five-dimensional gauge theories of different rank. We can also describe the five-dimensional theories that can be obtained from a six-dimensional $U(N)$ theory in terms of the parameter $N$. In this description, the possible five-dimensional theories include $\left[U\left(\frac{N+1}{D}\right)\right]^{D-1}$ with $D-2$ bifundamentals and $2\left(\frac{N+1}{D}-1\right)$ fundamentals, where $D$ is any positive nontrivial divisor of $N+1$. This presentation explains various gaps in Table I. Indeed, when $N+1$ is a prime number, there are no nontrivial divisors and, hence, no five-dimensional limit of the type discussed above. 
TABLE I. Nontrivial five-dimensional limits for $N \leq 15$.

\begin{tabular}{rrrrcrr}
\hline \hline & \multicolumn{3}{c}{ Parameters of $X_{A+B+A B, 1}^{(\delta=B(A+1)-1)}$} & \multicolumn{3}{c}{ Five-dim. gauge theory } \\
\hline$N$ & $A$ & $B$ & $\delta=B(A+1)-1$ & Gauge group & $\mathbf{F}$ & $\mathbf{B F}$ \\
3 & 1 & 1 & 1 & $S U(2)$ & 2 & 0 \\
5 & 2 & 1 & 2 & $U(3)$ & 4 & 0 \\
& 1 & 2 & 3 & {$[U(2)]^{2}$} & 2 & 1 \\
7 & 3 & 1 & 3 & $U(4)$ & 6 & 0 \\
& 1 & 3 & 5 & {$[U(2)]^{3}$} & 2 & 2 \\
8 & 2 & 2 & 5 & {$[U(3)]^{2}$} & 4 & 1 \\
9 & 4 & 1 & 4 & $U(5)$ & 8 & 0 \\
& 1 & 4 & 7 & {$[U(2)]^{4}$} & 2 & 3 \\
11 & 5 & 1 & 5 & $U(6)$ & 10 & 0 \\
& 3 & 2 & 7 & {$[U(4)]^{2}$} & 6 & 1 \\
& 2 & 3 & 8 & {$[U(3)]^{3}$} & 4 & 2 \\
& 1 & 5 & 9 & {$[U(2)]^{5}$} & 2 & 4 \\
13 & 6 & 1 & 6 & $U(7)$ & 12 & 0 \\
& 1 & 6 & 11 & {$[U(2)]^{6}$} & 2 & 5 \\
14 & 4 & 2 & 9 & {$[U(5)]^{2}$} & 8 & 1 \\
& 2 & 4 & 11 & {$[U(3)]^{4}$} & 4 & 3 \\
15 & 7 & 1 & 7 & $U(8)$ & 14 & 0 \\
& 3 & 3 & 11 & {$[U(4)]^{3}$} & 6 & 2 \\
& 1 & 7 & 13 & {$[U(2)]^{7}$} & 2 & 6 \\
\hline \hline
\end{tabular}

\section{CONCLUSIONS}

In this paper, we continued our exploration of the extended Kähler moduli space of a class of toric Calabi-Yau threefolds $X_{N, 1}$. In previous work [42], we have shown that the latter engineers a $U(N)$ gauge theory with adjoint matter on $\mathbb{R}^{5} \times S^{1}$, which in the limit of vanishing $S^{1}$ radius gives rise to a five-dimensional theory with the same gauge group. In this work, we investigated regions in the moduli space of $X_{N, 1}$, where the geometry is described by so-called shifted web diagrams $X_{N, 1}^{(\delta)}$ (with $\delta \in\{0, \ldots, N-1\}$ ) first introduced in [24], an example of which is shown in Fig. 1. We specifically investigated codimension-one limits in which the latter engineer five-dimensional quiver gauge theories with gauge group $G \subset U(N)$ (and various different matter contents). At the level of the six-dimensional theories, the topological string partition functions computed from $X_{N, 1}^{(\delta)}$ and $X_{N, 1}^{(0)}=X_{N, 1}$ are identical (as shown in [26]) and, thus, engineer dual six-dimensional gauge theories, which, however, admit different five-dimensional limits.

Our analysis is based on a number of specific examples, from which we managed to extract an emergent pattern, which we conjecture to hold in general: Starting from a sixdimensional $\mathcal{N}=(1,0) U(N)$ gauge theory with adjoint matter, we conjecture that there exists a five-dimensional limit engineering an $\mathcal{N}=1\left[U\left(\frac{N+1}{D}\right)\right]^{D-1}$ gauge theory with matter transforming in $D-2$ copies of the bifundamental and $2\left(\frac{N+1}{D}-1\right)$ copies of the fundamental representation, where $D$ is a positive nontrivial divisor of $N+1$. Furthermore, the limits can already be described at the level of the web diagram, which allows for a direct computation of the partition function using the (refined) topological vertex (as showcased in the example $X_{3,1}^{(1)}$, where the fivedimensional limit gives rise to the web diagram of $d P_{3}$ ).

The limits we studied in this paper certainly do not exhaust all possible ways to construct five-dimensional gauge theories from parent theories on $\mathbb{R}^{5} \times S^{1}$ that are engineered from (shifted) web diagrams. As an example, several webs allow for limits in which the six-dimensional gauge group $U(N)$ is completely broken to $[U(1)]^{N}$, which, however, were not discussed in the present work, since they are rather trivial. We, however, cannot rule out the existence of further limits which give rise to five-dimensional limits with a nontrivial gauge group and leave the study of their existence to future work. Conversely, the five-dimensional theories obtained in this work are consistent with the classification of five-dimensional theories discussed in [5], as all of them appear via geometric engineering from Calabi-Yau threefolds.

\section{ACKNOWLEDGMENTS}

A. I. thanks the hospitality of the Simons Center for Geometry and Physics during the Summer Workshop in Mathematics and Physics.

\section{APPENDIX A: DECOMPACTIFYING LIMIT AND THE PARTITION FUNCTION}

In this Appendix, we discuss the relation between taking the limit $Q_{\star} \mapsto 0$ (at the level of the partition function $\mathcal{Z}_{N, M}$ ) and the corresponding effect on the web diagram of $X_{N, M}^{(\delta)}$ (with a potential shift $\delta \neq 0$ ). We shall keep the discussion completely generic and consider $Q_{\star}=e^{-t_{\star}}$, where $t_{\star}$ is the Kähler parameter such that (keeping all other parameters fixed) it controls the size of a certain number of $\mathbb{P}^{1}$ 's in the geometry. We would like to show that in the limit $Q_{\star} \mapsto 0$ the topological string partition function remains well defined and is given by a corresponding decompactification of the web diagram. Let $W$ be the web diagram, and denote the corresponding partition function by $Z_{W}$. In the limit $Q_{\star} \mapsto 0$, suppose that the web diagram becomes $W_{0}$ with partition function $Z_{W_{0}}$. Recall that the topological string partition function written with the help of the topological vertex is an expansion near the large Kähler structure limit [43] and is given by a power series in $e^{-t_{a}}$, where $t_{a}$ are the Kähler parameters. In the case at hand, we keep the Kähler parameters other than $t_{\star}$ fixed as we take the limit $t_{\star} \mapsto \infty$.

We consider the partition function $Z_{W} / Z_{W_{0}}$ as an expansion in $Q_{\star}$ :

$$
\begin{aligned}
& \frac{Z_{W}}{Z_{W_{0}}} \sum_{\nu_{1} \ldots \nu_{k}} Q_{\star}^{\left|\nu_{1}\right|+\cdots+\left|\nu_{k}\right|} \frac{Z_{\nu_{1} \ldots \nu_{k}}\left(Q_{i}, q, t\right)}{Z_{W_{0}}} \\
& \quad=\sum_{K \geq 0} Q_{\star}^{K} \frac{W_{K}\left(Q_{i}, q, t\right)}{Z_{W_{0}}}=1+Q_{\star} \frac{W_{1}}{Z_{W_{0}}}+\cdots,
\end{aligned}
$$


with the expansion coefficients

$$
W_{K}\left(Q_{i}, q, t\right)=\sum_{\left|\nu_{1}\right|+\cdots+\left|\nu_{k}\right|=K} Z_{\nu_{1} \ldots \nu_{k}} .
$$

The function $Z_{\nu_{1} \ldots \nu_{k}}$ is the open string partition function which captures the contribution of open strings in the presence of Lagrangian branes with boundary conditions given by $\left\{\nu_{1}, \ldots, \nu_{k}\right\}[44,45]$. These Lagrangian branes are placed on the legs which get decompactified as $Q_{\star} \mapsto 0$. The function $W_{K}$ does not depend on $Q_{\star}$ and is well defined for any $K$. It captures the DT invariants [46] for a class of sheaves supported on the curves labeled by the class of $Q_{\star}$. Since $W_{K}$ do not depend on $Q_{\star}$, in the limit $Q_{\star} \mapsto 0$ the partition function $Z_{W}$ reduces to $Z_{W_{0}}$.

\section{APPENDIX B: INTERSECTION NUMBERS FOR $X_{N, M}$}

In the following, we give a very rough calculation of the intersection numbers in the elliptic Calabi-Yau threefold $X_{N, M}^{(\delta)}$, relying mostly on known results in the literature.

\section{Infinite toric fan}

As in [23], we start by considering an infinite toric fan, which can be decomposed into the following set of maximal cones in $\mathbb{R}^{3}$ :

$\sigma_{i, j}^{1}=\mathbb{R}_{\geq 0}(i, j, 1)+\mathbb{R}_{\geq 0}(i+1, j, 1)+\mathbb{R}_{\geq 0}(i, j+1,1)$,

$\sigma_{i, j}^{2}=\mathbb{R}_{\geq 0}(i+1, j, 1)+\mathbb{R}_{\geq 0}(i, j+1,1)+\mathbb{R}_{\geq 0}(i+1, j+1,1)$,

$$
i, j \in \mathbb{Z},
$$

where the triples $(i, j, k) \in \mathbb{Z}^{3}$ are called ray generators in the following. Since all ray generators in (B1) end on the same plane defined by $z=1$ in $\mathbb{R}$ (i.e., $k=1$ in all cases), the resulting geometry is Calabi-Yau and noncompact. A local region of the toric fan looks as shown in Fig. 13, where it is sufficient to show only the $x-y$ plane at $z=1$ due to the Calabi-Yau condition. Each wall, that is the intersection of two maximal cones, defines an irreducible toric curve. There are three families of curves, diagonal (blue), horizontal (green), and vertical (red) [the orientations are defined with respect to the dual toric graph Fig. 13(b)]. The walls have the following form:

$$
\begin{aligned}
\tau_{i, j} & =\sigma_{i, j}^{1} \cap \sigma_{i, j}^{2}=\mathbb{R}_{\geq 0}(i+1, j, 1)+\mathbb{R}_{\geq 0}(i, j+1,1), \\
\tau_{i+1, j} & =\sigma_{i, j}^{2} \cap \sigma_{i+1, j}^{1}=\mathbb{R}_{\geq 0}(i+1, j, 1)+\mathbb{R}_{\geq 0}(i+1, j+1,1), \\
\tau_{i, j+1} & =\sigma_{i, j}^{2} \cap \sigma_{i, j+1}^{1}=\mathbb{R}_{\geq 0}(i, j+1,1)+\mathbb{R}_{\geq 0}(i+1, j+1,1) .
\end{aligned}
$$

Because of $S L(2, \mathbb{Z})$ symmetry, the three families of curves are equivalent to each other; i.e., they are mapped into

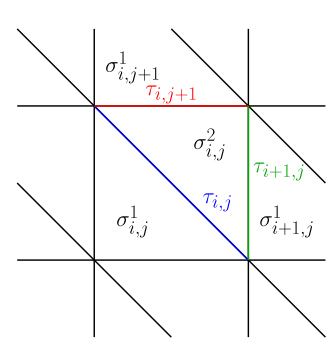

(a)

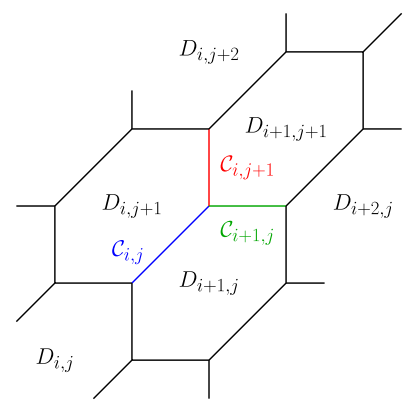

(b)
FIG. 13. (a) A local view of the $x-y$ plane at $z=1$ of an infinite toric fan with some maximal cones $\sigma$ and walls $\tau$ labeled. (b) A local view of the dual diagram to our infinite fan with some curves and divisors labeled.

another in different $S L(2, \mathbb{Z})$ frames. It is thus sufficient to focus on one class of curves. We choose the diagonal (blue) one. In the following, we shall follow [47] for a general result (reduced to a three-dimensional fan) and apply it directly to the specific construction above: Let $u_{i=0,1,2,3}$ be four ray generators in a smooth three-dimensional toric fan. If $\tau=\sigma \cap \sigma^{\prime}$ is a wall in the latter, which is defined through

$$
\begin{aligned}
\tau & =\mathbb{R}_{\geq 0} u_{1}+\mathbb{R}_{\geq 0} u_{2}, \quad \sigma=\mathbb{R}_{\geq 0} u_{0}+\mathbb{R}_{\geq 0} u_{1}+\mathbb{R}_{\geq 0} u_{2}, \\
\sigma^{\prime} & =\mathbb{R}_{\geq 0} u_{1}+\mathbb{R}_{\geq 0} u_{2}+\mathbb{R}_{\geq 0} u_{3},
\end{aligned}
$$

there exist integers $b_{1,2}$ such that the wall relation

$$
u_{0}+b_{1} u_{1}+b_{2} u_{2}+u_{3}=0
$$

is satisfied. The intersection number of the irreducible curve $\mathcal{C}_{\tau}$ associated to $\tau$ with the divisor $D_{u}$ associated to any ray generator $u$ of the fan is then given by

$$
D_{u} \cdot \mathcal{C}_{\tau}= \begin{cases}1 & \text { if } u=u_{0}, u_{3} \\ b_{i} & \text { if } u=u_{i} \text { for } i=1,2 \\ 0 & \text { else }\end{cases}
$$

Specifically, for the toric fan (B1), we have the wall relation for $\tau_{i, j}$ defined in (B2):

$$
\begin{aligned}
& (i, j, 1)-1(i+1, j, 1)-1(i, j+1,1)+(i+1, j+1,1)=0, \\
& \text { with } b_{1}=b_{2}=-1 .
\end{aligned}
$$

Thus, (B4) directly yields the following intersection numbers for the curves associated to $\tau_{i, j}$ in (B2), with all divisors (associated with the ray generators $u$ ): 


$$
D_{u} \cdot \mathcal{C}_{\tau_{i, j}}= \begin{cases}1 & \text { if } u=u_{0}, u_{3}, \\ -1 & \text { if } u=u_{k} \text { for } k=1,2 \\ 0 & \text { else. }\end{cases}
$$

By $S L(2, \mathbb{Z})$ symmetry, we thus have the following nonzero intersection numbers for the dual toric diagram in Fig. 13(b):

$$
\begin{gathered}
D \cdot \mathcal{C}_{i, j}= \begin{cases}1 & \text { if } D=D_{i, j}, D_{i+1, j+1}, \\
-1 & \text { if } D=D_{i+1, j}, D_{i, j+1},\end{cases} \\
D \cdot \mathcal{C}_{i+1, j}= \begin{cases}1 & \text { if } D=D_{i, j+1}, D_{i+2, j}, \\
-1 & \text { if } D=D_{i+1, j}, D_{i+1, j+1},\end{cases} \\
D \cdot \mathcal{C}_{i, j+1}= \begin{cases}1 & \text { if } D=D_{i+1, j}, D_{i, j+2}, \\
-1 & \text { if } D=D_{i, j+1}, D_{i+1, j+1}\end{cases}
\end{gathered}
$$

Summarizing these results in words, we can say: The intersection of a curve $\mathcal{C}$ with a divisor $D$ is 1 if $\mathcal{C}$ sticks out $D$, and it is -1 if $\mathcal{C}$ lies inside $D$.

\section{2. $X_{N, M}^{(\delta)}$ and intersection numbers}

In [23], the authors gave a toric construction of $X_{N, M}$. Roughly speaking, they consider an infinite toric fan quotiented by $N \mathbb{Z} \times M \mathbb{Z}$ to impose periodic identifications in the web diagram. In the following, we assume ${ }^{8}$ that there exists a similar quotient, which gives rise to the periodic identifications required in the web diagram $X_{N, M}^{(\delta)}$. For most curves and divisors, this quotient does not change the intersection numbers as devised in the previous section.

\footnotetext{
${ }^{8}$ Although we have not checked explicitly the existence of a quotient that satisfies all consistency conditions given in [23], we have checked our results for the intersection numbers (B8) and (B9) in various cases through other methods. In particular, for the cases $X_{2,1}^{(\delta)}, X_{2,2}^{(\delta)}($ for $\delta=0,1)$ and $X_{2, N}^{(\delta)}($ for $\delta=0,1, \ldots, N-1$ ) we have calculated them independently by representing the geometry locally as (combinations of) $\mathbb{P}^{1} \times \mathbb{P}^{1}$ and found complete agreement. This leads us to believe that a quotient procedure as detailed below can be employed to compute the intersection numbers.
}

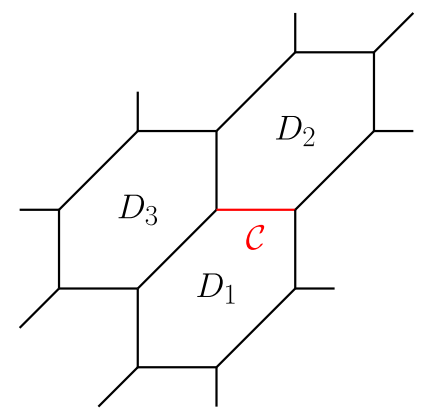

FIG. 14. A curve $\mathcal{C}$ and divisors $D_{1}, D_{2}$, and $D_{3}$.

Nevertheless, in the webs $X_{N, M}^{(\delta)}$ with $N=1$ or $M=1$, some curves will see their intersections numbers modified due to the fact that a given curve $\mathcal{C}$ can now intersect two irreducible divisors $D$ and $D^{\prime}$ that are identified under the quotient procedure. We will discuss in the following the two special situations that may arise for $N$ or $M$ equal to 1 , thus changing the effective rule given at the end of the previous section. It is sufficient to focus on configurations of type $X_{N, 1}$, as the $X_{1, M}$ configuration will follow the same pattern by $S L(2, \mathbb{Z})$ symmetry.

(i) Case 1.-The curve $\mathcal{C}$ lies inside two divisors which get identified under the quotient action. In the infinite fan, we can consider the intersection of the toric curve $\mathcal{C}$ with the divisor $D=D_{1}+D_{2}$ in Fig. 14:

$$
\mathcal{C} \cdot D=\mathcal{C} \cdot D_{1}+\mathcal{C} \cdot D_{2}=1+1=2 .
$$

Under the quotient action, the two irreducible divisors get identified $D_{1} \sim D_{2}$, leading to the result above.

(ii) Case 2.-The curve $\mathcal{C}$ lies in one divisor and sticks out of another one, and both get identified under the quotient. In terms of the infinite fan, we are interested in the intersection of $\mathcal{C}$ with the divisor $D^{\prime}=D_{1}+D_{3}$ (see Fig. 14):

$$
\mathcal{C} \cdot D^{\prime}=\mathcal{C} \cdot D_{1}+\mathcal{C} \cdot D_{3}=1-1=0 .
$$

[1] J. J. Heckman, D. R. Morrison, and C. Vafa, On the classification of 6D SCFTs and generalized ADE orbifolds, J. High Energy Phys. 05 (2014) 028; Erratum, J. High Energy Phys. 06 (2015) 017(E).

[2] J. J. Heckman, D. R. Morrison, T. Rudelius, and C. Vafa, Atomic classification of 6D SCFTs, Fortschr. Phys. 63, 468 (2015).
[3] M. Del Zotto, J. J. Heckman, and D. R. Morrison, 6D SCFTs and phases of 5D theories, J. High Energy Phys. 09 (2017) 147.

[4] P. Jefferson, H. C. Kim, C. Vafa, and G. Zafrir, Towards classification of 5d SCFTs: Single gauge node, arXiv: 1705.05836 . 
[5] P. Jefferson, S. Katz, H. C. Kim, and C. Vafa, On geometric classification of 5d SCFTs, J. High Energy Phys. 04 (2018) 103.

[6] L. Bhardwaj, M. Del Zotto, J. J. Heckman, D. R. Morrison, T. Rudelius, and C. Vafa, F-theory and the classification of little strings, Phys. Rev. D 93, 086002 (2016).

[7] N. Seiberg and E. Witten, Comments on string dynamics in six-dimensions, Nucl. Phys. B471, 121 (1996).

[8] M. Berkooz, M. Rozali, and N. Seiberg, Matrix description of M theory on $T^{4}$ and $T^{5}$, Phys. Lett. B 408, 105 (1997).

[9] J.D. Blum and K.A. Intriligator, New phases of string theory and 6-D RG fixed points via branes at orbifold singularities, Nucl. Phys. B506, 199 (1997).

[10] N. Seiberg, New theories in six-dimensions and matrix description of $\mathrm{M}$ theory on $T^{5}$ and $T^{5} / Z_{2}$, Phys. Lett. B 408, 98 (1997).

[11] A. Losev, G. W. Moore, and S. L. Shatashvili, M \& m's, Nucl. Phys. B522, 105 (1998).

[12] K. A. Intriligator, New string theories in six-dimensions via branes at orbifold singularities, Adv. Theor. Math. Phys. 1, 271 (1997).

[13] O. Aharony, M. Berkooz, D. Kutasov, and N. Seiberg, Linear dilatons, NS five-branes and holography, J. High Energy Phys. 10 (1998) 004.

[14] O. Aharony, A brief review of "little string theories", Classical Quantum Gravity 17, 929 (2000).

[15] D. Kutasov, Introduction to Little String Theory, in Superstrings and Related Matters. Proceedings, Spring School, Trieste, Italy, 2001 (World Scientific Pub Co Inc., Singapore, 2001), pp. 165-209.

[16] E. Witten, Phase transitions in M theory and F theory, Nucl. Phys. B471, 195 (1996).

[17] D. R. Morrison and C. Vafa, Compactifications of F theory on Calabi-Yau threefolds. 1, Nucl. Phys. B473, 74 (1996).

[18] D. R. Morrison and C. Vafa, Compactifications of F theory on Calabi-Yau threefolds. 2, Nucl. Phys. B476, 437 (1996).

[19] A. S. Chou, R. Kallosh, J. Rahmfeld, S. J. Rey, M. Shmakova, and W. K. Wong, Critical points and phase transitions in 5-D compactifications of $\mathrm{M}$ theory, Nucl. Phys. B508, 147 (1997).

[20] B. Haghighat, A. Iqbal, C. Kozaz, G. Lockhart, and C. Vafa, M-strings, Commun. Math. Phys. 334, 779 (2015).

[21] B. Haghighat, C. Kozcaz, G. Lockhart, and C. Vafa, Orbifolds of M-strings, Phys. Rev. D 89, 046003 (2014).

[22] S. Hohenegger and A. Iqbal, M-strings, elliptic genera and $\mathcal{N}=4$ string amplitudes, Fortschr. Phys. 62, 155 (2014).

[23] A. Kanazawa and S. C. Lau, Local Calabi-Yau manifolds of type $\tilde{A}$ and open Yau-Zaslow formula via SYZ mirror symmetry, arXiv:1605.00342v2.

[24] S. Hohenegger, A. Iqbal, and S. J. Rey, Dual little strings from F-theory and flop transitions, J. High Energy Phys. 07 (2017) 112.

[25] B. Bastian, S. Hohenegger, A. Iqbal, and S. J. Rey, Beyond triality: Dual Quiver gauge theories and little string theories, J. High Energy Phys. 11 (2018) 016.

[26] B. Bastian, S. Hohenegger, A. Iqbal, and S. J. Rey, Dual little strings and their partition functions, Phys. Rev. D 97, 106004 (2018).
[27] M. Reid, The moduli space of 3-folds with $K=0$ may nevertheless be irreducible, Math. Ann. 278, 329 (1987).

[28] P. S. Green and T. Hübsch, Connecting moduli spaces of Calabi-Yau threefolds, Commun. Math. Phys. 119, 431 (1988).

[29] P. S. Green and T. Hübsch, Phase Transitions Among (Many of) Calabi-Yau Compactifications, Phys. Rev. Lett. 61, 1163 (1988).

[30] P. Candelas, P. S. Green, and T. Hübsch, Finite Distances between Distinct Calabi-Yau Vacua: (Other Worlds Are Just around the Corner), Phys. Rev. Lett. 62, 1956 (1989).

[31] P. Candelas, P. S. Green, and T. Hübsch, Rolling among Calabi-Yau Vacua, Nucl. Phys. B330, 49 (1990).

[32] S. Hohenegger, A. Iqbal, and S. J. Rey, Self-duality and selfsimilarity of little string orbifolds, Phys. Rev. D 94, 046006 (2016).

[33] B. Bastian, S. Hohenegger, A. Iqbal, and S. J. Rey, Triality in little string theories, Phys. Rev. D 97, 046004 (2018).

[34] M. X. Huang, S. Katz, and A. Klemm, Topological string on elliptic CY 3-folds and the ring of Jacobi forms, J. High Energy Phys. 10 (2015) 125.

[35] A. Klemm, J. Manschot, and T. Wotschke, Quantum geometry of elliptic Calabi-Yau manifolds, Commun. Number Theor. Phys. 6, 849 (2012).

[36] O. Aharony, A. Hanany, and B. Kol, Webs of (p,q) fivebranes, five-dimensional field theories and grid diagrams, J. High Energy Phys. 01 (1998) 002.

[37] M. Taki, Seiberg duality, 5d SCFTs and Nekrasov partition functions, arXiv:1401.7200.

[38] N. A. Nekrasov, Seiberg-Witten prepotential from instanton counting, Adv. Theor. Math. Phys. 7, 831 (2003).

[39] H. Nakajima and K. Yoshioka, Instanton counting on blowup. II. K-theoretic partition function, arXiv:math/ 0505553.

[40] H. Awata and Y. Yamada, Five-dimensional AGT relation and the deformed beta-ensemble, Prog. Theor. Phys. 124, 227 (2010).

[41] M. Esole and S. H. Shao, M-theory on Elliptic Calabi-Yau threefolds and 6d anomalies, arXiv:1504.01387.

[42] S. Hohenegger, A. Iqbal, and S. J. Rey, Instanton-monopole correspondence from M-branes on $\mathbb{S}^{1}$ and little string theory, Phys. Rev. D 93, 066016 (2016).

[43] M. Bershadsky, S. Cecotti, H. Ooguri, and C. Vafa, Kodaira-Spencer theory of gravity and exact results for quantum string amplitudes, Commun. Math. Phys. 165, 311 (1994).

[44] M. Aganagic and C. Vafa, Mirror symmetry, D-branes and counting holomorphic discs, arXiv:hep-th/0012041.

[45] M. Aganagic, A. Klemm, M. Marino, and C. Vafa, The topological vertex, Commun. Math. Phys. 254, 425 (2005).

[46] D. Maulik, N. Nekrasov, A. Okounkov, and R. Pandharipande, Gromov-Witten theory and Donaldson-Thomas theory, I, arXiv:math/0312059.

[47] D. Cox, J. Little, and H. Schenck, Toric Varieties (American Mathematical Society, Providence, RI, 2010). 